\title{
Pitch and Thrust Allocation for Full-Flight-Regime Control of Winged eVTOL UAVs
}

Jacob B. Willis

Brigham Young University, jbwillis272@gmail.com

Randal W. Beard

Brigham Young University, beard@byu.edu

Follow this and additional works at: https://scholarsarchive.byu.edu/facpub

Part of the Electrical and Computer Engineering Commons

\section{BYU ScholarsArchive Citation}

Willis, Jacob B. and Beard, Randal W., "Pitch and Thrust Allocation for Full-Flight-Regime Control of Winged eVTOL UAVs" (2020). Faculty Publications. 5212.

https://scholarsarchive.byu.edu/facpub/5212

This Conference Paper is brought to you for free and open access by BYU ScholarsArchive. It has been accepted for inclusion in Faculty Publications by an authorized administrator of BYU ScholarsArchive. For more information, please contact ellen_amatangelo@byu.edu. 


\title{
Pitch and Thrust Allocation for Full-Flight-Regime Control of Winged eVTOL UAVs
}

\author{
Jacob B. Willis ${ }^{1}$ and Randal W. Beard ${ }^{2}$
}

\begin{abstract}
Trajectory tracking control for winged eVTOL aircraft is complicated by the high-angle-of-attack aerodynamics experienced during navigational flight occurring immediately after takeoff and immediately before landing. The total energy use of the vehicle can be reduced and the control performance can be improved by appropriately considering the pitch angle of the vehicle in varying flight conditions. We present a review of high-angle-of-attack aerodynamic models as well as an algorithm for finding the optimal pitch and thrust of a winged eVTOL throughout its flight regime. We show simulation results demonstrating a $75 \%$ reduction in tracking error over our previous work while maintaining a similar average thrust and an $\mathbf{8 5 \%}$ reduction in tracking error over using a multirotor-like controller.
\end{abstract}

\section{INTRODUCTION}

One of the major challenges in the control design of winged electric vertical takeoff and landing (eVTOL) unmanned aerial vehicles (UAVs) is the high-angle-of-attack aerodynamics they experience while in partially transitioned flight regimes. Because of the nature of turbulent flow, these aerodynamics are challenging to predict and will likely vary with minor changes in flight conditions.

In our previous work [1], we develop a trajectory tracking controller that can be generally applied to any vectored thrust or lift and cruise winged eVTOL UAVs 1 . We demonstrate that, within the constraints of the thrust vector, trajectory tracking can be performed at any pitch angle. The pitch angle is then a free variable that can be used to satisfy secondary objectives such as minimizing thrust or ensuring passenger comfort. We refer to finding the appropriate pitch angle, and the related thrust vector, as the pitch and thrust allocation problem. Figure 1 shows the overall architecture of our trajectory tracking controller and highlights how solving the pitch and thrust allocation problem fits into the complete control system.

In [1] we solve the pitch and thrust allocation problem using a general-purpose nonlinear optimizer. The shortcomings of this approach, however, are the optimizer cannot handle the problem's multiple local minima and the high computational cost of an optimizer embedded within a control loop. Here, we investigate the pitch allocation problem in more

\footnotetext{
${ }^{1}$ Graduate research assistant, BYU MAGICC lab.

${ }^{2}$ Professor of Electrical and Computer Engineering, Brigham Young University, beardabyu . edu

*This work has been funded by the Center for Unmanned Aircraft Systems (C-UAS), a National Science Foundation Industry/University Cooperative Research Center (I/UCRC) under NSF award No. IIP-1650547, along with significant contributions from C-UAS industry members.

${ }^{1}$ See https://evtol.news/classifications
}

detail; we review post-stall aerodynamic models found in the literature and propose a search-based method for finding the global optimum of the pitch allocation problem. We also point out that while the pitch allocation problem exhibits multiple local minima that vary with the optimization parameters, the locations of the minima vary slowly, so we propose an iterative method for refining the optimal solution over multiple iterations of the control loop.

Previous work in this area is limited. The work on trajectory tracking for winged eVTOLs by Anglade, et al. [2] utilizes a flat-plate lift and drag model and finds an analytic solution to the pitch and thrust allocation problem. However, the solution ignores constraints on the desired pitch angle and does not apply to alternative lift-drag models, such as those discussed in section II. A similar control architecture for multirotors is the controller by Lee, Leok, and McClamroch [3]. Because multirotors lack lifting surfaces and thrust vectoring, the roll and pitch of the vehicle are both defined by pointing the $-\boldsymbol{z}_{b}$ axis in the direction of the desired force vector. This method of determining the pitch can also be applied to winged eVTOLs by ignoring aerodynamic forces and vectored thrust and is the only mode available for lowspeed winged eVTOL control in the PX4 Autopilot [4]. We use it as a baseline comparison in our results.

We begin in section III by comparing multiple highangle-of-attack lift and drag models found in the literature. We then cover the pitch and thrust allocation problem in section III and in section IV we show the trajectory tracking improvements of our new algorithm over using a nonlinear optimizer as done in our previous work [1].

Throughout this paper the notation $\boldsymbol{r}_{a / b}^{c}$ is used to denote a vector quantity $\boldsymbol{r}$ of frame $a$ with respect to frame $b$ and expressed in frame $c$. Similarly, the notation $\varphi_{a / b}$ is used for an angle from frame $b$ to frame $a$. We use $i$ to refer to the north-east-down inertial frame, and $b$ to refer to the true body-fixed frame. The desired, desired pitch, and stability frames, are denoted $d, p$, and $s$ respectively. The inputs to the pitch and thrust allocation block in fig. 1 are expressed in the desired frame, which has been rolled and yawed such that the commanded force vector resides in the vehicle longitudinal plane. The outputs of the pitch and thrust allocation are expressed in the desired pitch frame. The stability frame is used to express the aerodynamic forces and is described in more detail subsequently. 


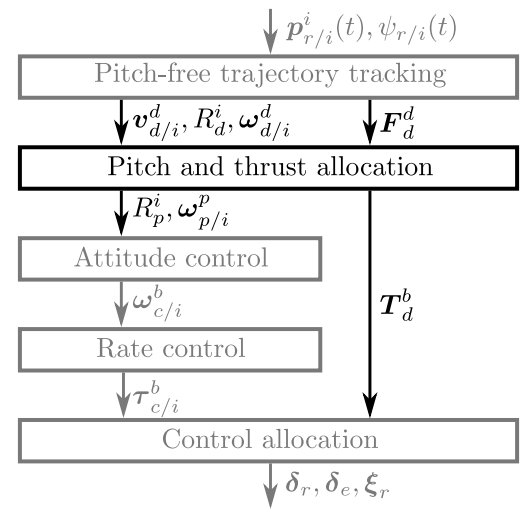

Fig. 1. Block diagram of the control scheme we presented in [1]. The controller tracks a three times differentiable position trajectory and a one time differentiable yaw trajectory. This paper focuses on the emphasized pitch and thrust allocation block.

TABLE I

AERODYNAMIC AND VEHICLE MODEL NOMENCLATURE

\begin{tabular}{lcl}
\hline Symbol & Value & Description \\
\hline \hline$\alpha$ & - & Angle-of-attack (deg) \\
$\alpha_{0}$ & $15^{\circ}$ & Stall angle-of-attack \\
$C_{L_{0}}$ & 0.005 & Constant lift coefficient \\
$C_{L_{\alpha}}$ & 2.819 & Linear lift coefficient \\
$C_{D_{p}}$ & 0.003 & Parasitic drag coefficient \\
$A R$ & 7.815 & Wing aspect ratio \\
$S$ & 0.259 & Wing planform area $\left(\mathrm{m}^{2}\right)$ \\
$\rho$ & 1.268 & Air density $\left(\mathrm{kg} / \mathrm{m}^{3}\right)$ \\
$e$ & 0.9 & Oswald efficiency factor \\
$M$ & 50 & Blending transition rate \\
$m$ & 1.0 & Mass of vehicle $(\mathrm{kg})$ \\
$g$ & 9.81 & Acceleration due to gravity $\left(\mathrm{m} / \mathrm{s}^{2}\right)$ \\
$\xi_{\min }$ & $0^{\circ}$ & Minimum thrust angle \\
$\xi_{\max }$ & $90^{\circ}$ & Maximum thrust angle \\
$\theta_{\min }$ & $0^{\circ}$ & Minimum desired pitch angle \\
$\theta_{\max }$ & $15^{\circ}$ & Maximum desired pitch angle \\
& &
\end{tabular}

\section{High-Angle-OF-ATtACK Lift AND DRAg Models}

Table I lists the symbols used in this section, as well as the value of constants used throughout the paper. The vehicle-specific constants were determined for the E-Flight convergence aircraft, as described in [5].

At small angles of attack $\left(|\alpha|<\alpha_{0}\right)$, the behavior of lifting surfaces is well understood and typically modeled by a linear lift curve and a quadratic drag curve,

$$
\begin{aligned}
& C_{L, \mathrm{SA}}(\alpha)=C_{L_{0}}+C_{L_{\alpha}} \alpha \\
& C_{D, \mathrm{SA}}(\alpha)=C_{D_{p}}+\frac{\left(C_{L_{0}}+C_{L_{\alpha}} \alpha\right)^{2}}{\pi e A R} .
\end{aligned}
$$

However, as $|\alpha| \gg 0$, these models fail to predict the aerodynamic behavior of the wing. In their work on poststall perching, Cory and Tedrake [6] use a flat-plate model to fit flight data taken over the $\alpha \in\left[-20^{\circ}, 140^{\circ}\right]$ range. A similar model is proposed by [7] and applied to the control of a winged eVTOL in [2]. This model is equivalent in lift and adds a constant offset in drag to [6] and is given by

$$
\begin{aligned}
C_{L, \mathrm{FP} 1} & =2 \sin \alpha \cos \alpha \\
C_{D, \mathrm{FP} 1} & =C_{D_{p}}+2 \sin ^{2} \alpha .
\end{aligned}
$$

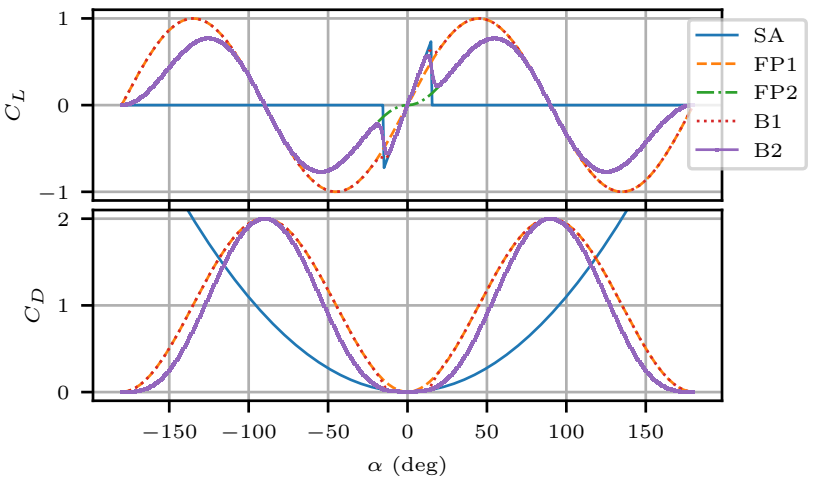

Fig. 2. Comparison of various high-angle-of-attack lift and drag models found in the literature. The small-angle models are good for $|\alpha| \leq \alpha_{0}$, the flat plate models are best for $|\alpha| \gg 0$, and the blended model combines the small-angle and flat plate model to cover a larger range of attack angles.

An alternative flat plate model is given by Stengel in [8],

$$
\begin{aligned}
C_{L, \mathrm{FP} 2} & =2 \operatorname{sgn}(\alpha) \sin ^{2} \alpha \cos \alpha \\
C_{D, \mathrm{FP} 2} & =2 \operatorname{sgn}(\alpha) \sin ^{3}(\alpha) .
\end{aligned}
$$

These flat plate models tend to underpredict lift for low angle-of-attack flight, as demonstrated in [9] where radial basis functions are fit to experimental data to produce a better $C_{L}$ match. An alternative, used in [10], [11], [12] is to blend the small-angle angle-of-attack lift and drag models in eq. (1) with the flat plate models in eq. (3). To blend the small-angle and flat plate models, we first define the sigmoid function,

$$
\sigma(\alpha)=\frac{1+e^{-M\left(\alpha-\alpha_{0}\right)}+e^{M\left(\alpha+\alpha_{0}\right)}}{\left(1+e^{-M\left(\alpha-\alpha_{0}\right)}\right)\left(1+e^{M\left(\alpha+\alpha_{0}\right)}\right)}
$$

where $M$ is the transition rate and $\alpha_{0}$ is the stall angle. Then the blended lift and drag coefficients are

$$
\begin{aligned}
C_{L, \mathrm{~B}^{*}} & =(1-\sigma(\alpha)) C_{L, \mathrm{SA}}(\alpha)+\sigma(\alpha) C_{L, \mathrm{FP}^{*}}(\alpha) \\
C_{D, \mathrm{~B}^{*}} & =(1-\sigma(\alpha)) C_{D, \mathrm{SA}}(\alpha)+\sigma(\alpha) C_{D, \mathrm{FP}^{*}}(\alpha) .
\end{aligned}
$$

Figure 2 shows a comparison of these different models. The small-angle lift curve is truncated at $\pm \alpha_{0}$. The blended model adds additional complexity of a minimum between the peak following the linear lift curve and the peak of the flat plate curve.

A comparison of the $C_{L} / C_{D}$ efficiency of the various aerodynamic models is shown in fig. 3 The flat plate models both exhibit significant differences from the small-angle model, whereas the blended models closely follow the smallangle curve for low $\alpha$ and converge to the flat plate curves for large $\alpha$. Because the flat plate models poorly predict the efficiency at small attack angles, we expect their performance during level, high-speed flight to be poorer than the blended models.

The flat plate and blended models capture the fundamental behavior of a wing at high attack angles, and the blended models capture the laminar flow aerodynamics at low attack angles. The blended models show a rapid decrease in lift at $\alpha_{0}$, providing a model for stall. However, in real flight, 


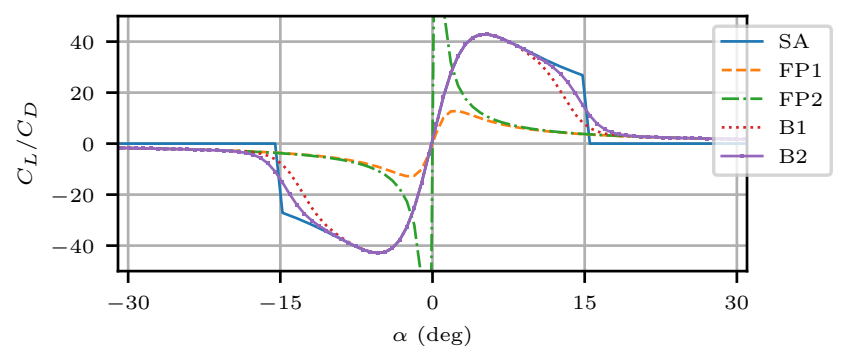

Fig. 3. Aerodynamic efficiency plot showing $C_{L} / C_{D}$ for $\alpha$ near 0. For $|\alpha|<\alpha_{0}=15^{\circ}$, the small-angle (SA) model is the best fit to a real aircraft.

small variations in the flight conditions would cause flow separation to occur at other angles. This loss of laminar flow exhibits hysteresis and has been previously modeled by incorporating a flow separation parameter in the system state [13], [14]. We do not investigate the use of a flow separation parameter in this work, however, the proposed solution to the pitch and thrust allocation problem can be applied to these models assuming the flow separation parameter is kept static on each iteration of the control loop.

\section{Pitch And Thrust Allocation}

The trajectory tracking controller given in our previous work [1] computes a desired applied force vector, $\boldsymbol{F}_{d}^{d}$, where $d$ signifies the desired frame. The desired frame is located at the center of mass of the vehicle and is rolled and yawed such that $\boldsymbol{F}_{d}^{d}$ lies in the body longitudinal plane, but the desired frame has a zero pitch angle. The task now remains to find the optimal pitch to achieve the desired applied forces through a combination of aerodynamic and propulsive forces. To do so, we find the pitch angle which minimizes the thrust necessary to achieve $\boldsymbol{F}_{d}^{d}$ by taking advantage of the aerodynamic forces on the vehicle while respecting limits on the thrust direction. We begin by describing the relationship between the total forces $\boldsymbol{F}_{d}^{d}$, the thrust $\boldsymbol{T}_{d}^{p}$ and the aerodynamic forces $\boldsymbol{F}_{\text {aero }}^{s}$. Recall that $p$ denotes the desired pitch frame, which is rotated about the $\boldsymbol{y}_{d}$ axis from the desired frame by $\theta_{p / d}$, and $s$ denotes the aerodynamic stability frame, where $\boldsymbol{y}_{s}=\boldsymbol{y}_{d}$ and $\boldsymbol{x}_{s}$ is aligned with the projection of the airspeed vector in the $\boldsymbol{x}_{d} \boldsymbol{z}_{d}$ plane. The lift and drag forces act along the $\boldsymbol{z}_{s}$ and $\boldsymbol{x}_{s}$ axes respectively. We assume zero wind, so the desired velocity vector $\boldsymbol{v}_{d / i}^{d}$ is the airspeed vector. The vector quantities and angles used in this section are depicted in fig. 4.

We let $V_{a}=\left\|\boldsymbol{v}_{d / i}^{d}\right\|$ be the magnitude of the desired airspeed. We define the flight path angle to be the angle between the horizontal plane and the desired velocity vector,

$$
\gamma_{s / d}=\tan ^{-1}\left(\frac{-\boldsymbol{v}_{z, d / i}^{d}}{\boldsymbol{v}_{x, d / i}^{d}}\right) .
$$

The stability frame is then defined as a rotation by $\gamma_{s / d}$ about the $\boldsymbol{y}_{d}$ axis. We define the angle-of-attack to be the angle between the $\boldsymbol{x}_{s}$ and $\boldsymbol{x}_{p}$ axes; because we assume wind is zero, $\theta_{p / d}=\alpha_{p / s}+\gamma_{s / d}$. We define the longitudinal aerodynamic force vector to be

$$
\boldsymbol{F}_{\text {aero }}^{s}\left(\alpha, V_{a}\right)=\left[\begin{array}{l}
-F_{\text {drag }}\left(\alpha, V_{a}\right) \\
-F_{\text {lift }}\left(\alpha, V_{a}\right)
\end{array}\right]
$$

where $F_{\text {lift }}\left(\alpha, V_{a}\right)=\frac{1}{2} \rho V_{a}^{2} S C_{L, *}(\alpha)$ and $F_{\text {drag }}\left(\alpha, V_{a}\right)=$ $\frac{1}{2} \rho V_{a}^{2} S C_{D, *}(\alpha)$, with $C_{L, *}$ and $C_{D, *}$ given by one of the models in section

We define the angle of the vectored thrust with respect to the pitched frame as

$$
\angle \boldsymbol{T}=\tan ^{-1}\left(\frac{-\boldsymbol{T}_{z, d}^{p}}{\boldsymbol{T}_{x, d}^{p}}\right),
$$

the angle of the desired force vector with respect to the desired frame as

$$
\angle \boldsymbol{F}=\tan ^{-1}\left(\frac{-\boldsymbol{F}_{z, d}^{d}}{\boldsymbol{F}_{x, d}^{d}}\right),
$$

and the desired force vector expressed in the stability frame as

$$
\boldsymbol{F}_{d}^{s}=\bar{R}\left(\gamma_{s / d}\right) \overline{\boldsymbol{F}}_{d}^{d}
$$

where

$$
\bar{R}(\varphi)=\left[\begin{array}{cc}
\cos \varphi & -\sin \varphi \\
\sin \varphi & \cos \varphi
\end{array}\right]
$$

is an active rotation by $\varphi$, and since $\boldsymbol{F}_{y, d}^{d}=0$ we define the two-vector $\overline{\boldsymbol{F}}_{d}^{d}=\left[\boldsymbol{F}_{x, d}^{d}, \boldsymbol{F}_{z, d}^{d}\right]$.

For the desired forces to be achieved, the applied thrust is a nonlinear function of $\theta_{p / d}$,

$$
\begin{aligned}
& \boldsymbol{T}_{d}^{p}\left(\theta_{p / d}\right)= \\
& \bar{R}\left(\theta_{p / d}-\gamma_{s / d}\right)\left(\boldsymbol{F}_{d}^{s}-\boldsymbol{F}_{\text {aero }}^{s}\left(\theta_{p / d}-\gamma_{s / d}, V_{a}\right)\right) . \\
& \underset{F_{\text {drag }}}{ }
\end{aligned}
$$

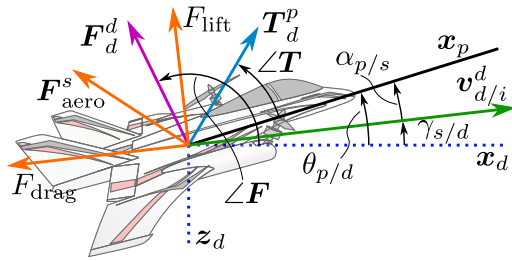

Fig. 4. Depiction of the vectors and angles used in computing the aerodynamic forces and thrust. The origin of frames $p$ and $s$ coincide with the origin of frame $d$. The $\boldsymbol{x}_{d}$ axis of the desired frame is in the horizontal plane, that is, it is orthogonal to the $\boldsymbol{z}_{i}$ axis of the inertial frame.

\section{A. Pitch and Thrust Optimization}

Through much of the flight regime, there is not one unique solution to eq. (12), so we use $\theta_{p / d}$ as an optimization 
variable to minimize the thrust magnitude, $\left\|\boldsymbol{T}_{d}^{p}\right\|$. The optimization is then

$$
\begin{array}{ll}
\text { given } & V_{a}, \gamma_{s / d}, \boldsymbol{F}_{d}^{s} \\
\min & \left\|\boldsymbol{T}_{d}^{p}\left(\theta_{p / d}\right)\right\|+\mu \theta_{p / d}^{2} \\
\text { w.r.t. } & \theta_{p / d} \\
\text { s.t. } & \boldsymbol{T}_{d}^{p}\left(\theta_{p / d}\right)= \\
& \bar{R}\left(\theta_{p / d}-\gamma_{s / d}\right)\left(\boldsymbol{F}_{d}^{s}-\boldsymbol{F}_{\text {aero }}^{s}\left(\theta_{p / d}-\gamma_{s / d}, V_{a}\right)\right) \\
& \theta_{p / d} \in\left[\theta_{\min }, \theta_{\max }\right] \\
& \angle \boldsymbol{T} \in\left[\xi_{\min }, \xi_{\max }\right] .
\end{array}
$$

Including $\mu \theta_{p / d}^{2}$ in the objective with $0<\mu \ll 1$ ensures a single minimum exists when $V_{a}=0$. The constraint on $\theta_{p / d}$ ensures the vehicle remains in a nominally level state, while the constraint on $\angle \boldsymbol{T}$ ensures the thrust is within the producible range. As given in eq. (13), the optimization will fail when the desired force vector is outside the range that is achievable while satisfying $\theta_{p / d} \in\left[\theta_{\min }, \theta_{\max }\right]$ and $\angle \boldsymbol{T} \in$ $\left[\xi_{\min }, \xi_{\max }\right]$. To accommodate this, we perform a second optimization when eq. (13) fails to find a feasible solution. The purpose of the secondary optimization in eq. (14) is to find a feasible thrust at pitch angles outside the desired range,

$$
\begin{array}{ll}
\text { given } & V_{a}, \gamma_{s / d}, \boldsymbol{F}_{d}^{s} \\
\min & \theta_{p / d}^{2} \\
\text { w.r.t. } & \theta_{p / d} \\
\text { s.t. } & \boldsymbol{T}_{d}^{p}\left(\theta_{p / d}\right)= \\
& \quad \bar{R}\left(\theta_{p / d}-\gamma_{s / d}\right)\left(\boldsymbol{F}_{d}^{s}-\boldsymbol{F}_{\text {aero }}^{s}\left(\theta_{p / d}-\gamma_{s / d}, V_{a}\right)\right) \\
& \theta_{p / d} \in\left[-\frac{\pi}{2},-\theta_{\min }\right] \cup\left[\theta_{\max }, \frac{\pi}{2}\right] \\
& \angle \boldsymbol{T} \in\left[\xi_{\min }, \xi_{\max }\right] .
\end{array}
$$

Because this searches outside the nominal range of pitch angles, we formulate the objective to select the feasible solution closest to satisfying the $\theta_{\min }, \theta_{\max }$ bounds rather than to select the minimum thrust solution. This enables the controller to handle scenarios such as fast reverse flight or rapid air braking where the vehicle uses the drag at a high angle of attack to quickly decelerate.

\section{B. Sampling-Based Pitch and Thrust Allocation}

Depending on the lift and drag model used, and because the angle-of-attack is frequently outside $\left[-\alpha_{0}, \alpha_{0}\right]$ eqs. (13) and (14) can have multiple local minima. We solve it globally on each iteration of the control loop by sampling and choosing the lowest cost feasible point. Because the input trajectory and system states vary continuously, the solution to eqs. (13) and (14) is similar from iteration to iteration. To avoid an excessively high number of samples in any one iteration, we increase the sampling density near the previous solution. The algorithm for computing the best pitch angle is shown in algorithm 11. Lines 1 through 5 solve eq. (13) and lines 6 through 9 solve eq. (14). Line 1 generates cubically spaced refinement samples around the previous optimal solution; it evaluates to \{\} when no previous solution exists.

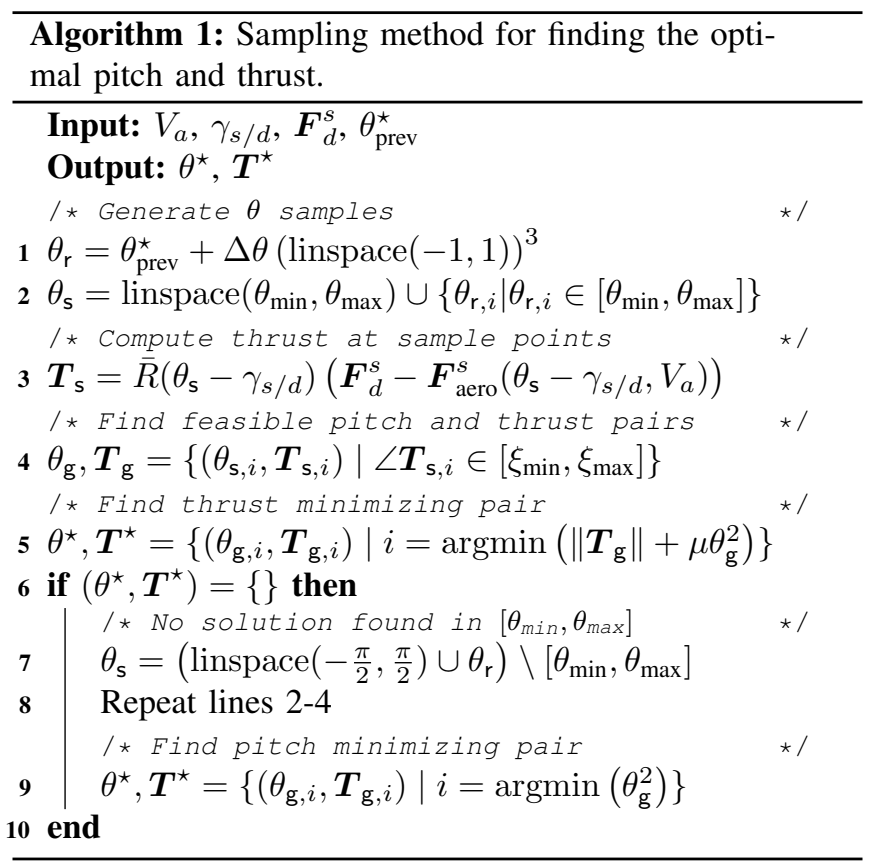

\section{Pitch Allocation Results}

Figures 5,6 and 7 show the result of the proposed pitch and thrust allocation algorithm along sweeps of varying inputs. In all figures, the small-angle with angle-of-attack cutoffs model (eq. (1)), the flat plate 1 model (eq. (2)), and the blended flat plate 2 (eq. (5) with eq. (3)) model are optimized using algorithm 1 and the legend entries for these models are SA, FP1, and $\mathrm{B} 2$ respectively. A comparison is made to using a multirotor-like method of pointing the $-\boldsymbol{z}_{p}$ axis in the direction of the desired force and computing the resulting thrust using eq. (12) and the blended flat plate 2 model. The legend entry for this method is $\angle \boldsymbol{F}_{d}^{d}$. Due to model differences, the absolute magnitude of thrust should only be compared between the optimized blended 2 and the multirotor blended 2 methods.

Figure 5 shows the effect of varying the flight path angles while the airspeed and desired force are held constant. The increase in $\theta^{\star}$ after $\gamma \approx 150^{\circ}$ is due to the first pitch allocation in eq. (13) failing and the second method in eq. (14) being applied. The discontinuity at this point is due to the wide spacing of the $\theta$ samples. It is resolved by subsequent iterations of the algorithm sampling near the previous solution. The pitch rate restriction described in eq. 15. prevents this behavior in the final control implementation. The sharp discontinuities in the small-angle plot are due to the truncation of the lift line. The discontinuities from $\theta^{\star}=\theta_{\max }$ to $\theta^{\star}=0$ near $\gamma=100^{\circ}$ is due to the blended and flat plate models exhibiting zero drag near $\alpha= \pm 180^{\circ}$.

Figure 6 shows the effect of increasing the airspeed while the flight path angle and desired force are held constant. This figure reflects a transition to forward flight, showing that at low airspeed, the pitch angle saturates at $\theta_{\max }$ and the thrust compensates for a lack of lift. As the vehicle accelerates, 
lift is produced by the wings, and near $V_{a}=10 \mathrm{~m} / \mathrm{s}$, it is no longer necessary to produce thrust along the $\boldsymbol{z}_{p}$ axis. This corresponds with a complete transition. Beyond $V_{a}=$ $10 \mathrm{~m} / \mathrm{s}, T_{x}$ increases slowly to compensate for the increasing parasitic drag. In comparison, the multirotor solution pitches the vehicle down to point the thrust in the direction of travel. As the vehicle accelerates the thrust required for the multirotor controller increases quadratically due to increasing drag. The comparison is cut off at $V_{a}=10 \mathrm{~m} / \mathrm{s}$ due to the unattainable thrust required at higher airspeed.

Figure 7 shows the effect of modifying the direction of the applied force while the flight path angle, airspeed, and $\boldsymbol{F}_{z, d}^{d}$ are held constant. For $F_{x}<-2.5$, the desired force vector cannot be achieved without violating the $\theta_{\max }$ bound, and the secondary optimization is used to find the appropriate pitch and thrust. Above this, the optimized methods stay at $\theta_{\max }$ and produce thrust using $T_{x}$ while the multirotor method continues to pitch forward, causing an increase in the total thrust magnitude.

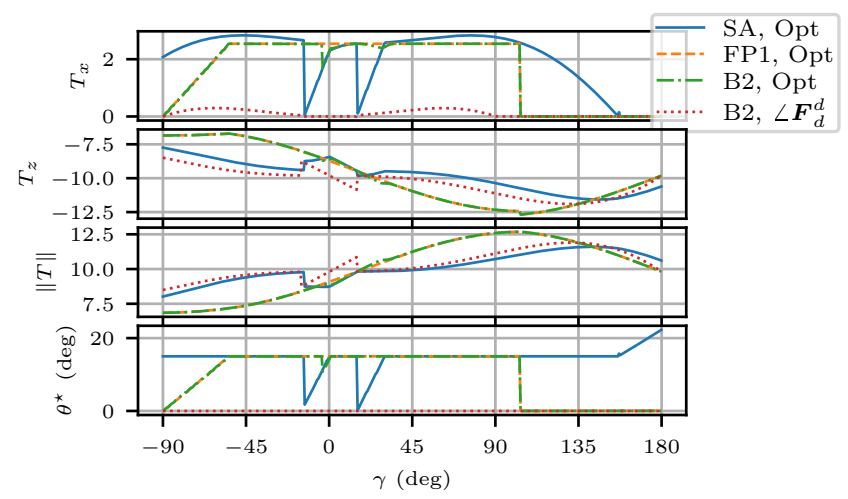

Fig. 5. Optimal thrust and pitch for increasing flight path angles. The other inputs to algorithm 1 are held constant at $V_{a}=3.0 \mathrm{~m} / \mathrm{s}$ and $F_{d}^{d}=$ $[0,0,-m g]^{T}$.

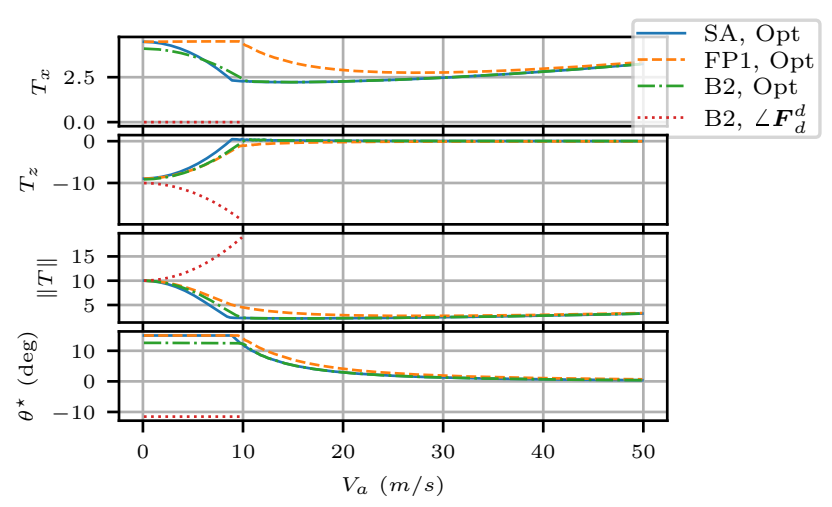

Fig. 6. Optimal thrust and pitch for increasing airspeed at a constant flight path angle. The other inputs to algorithm 1 are held constant at $\gamma=0^{\circ}$ and $\boldsymbol{F}_{d}^{d}=[2,0,-m g]^{T}$.

\section{INCORPORATION INTO FULL CONTROLLER}

To illustrate the improvements of using algorithm 1 to solve the pitch and thrust allocation optimization in eqs. (13)

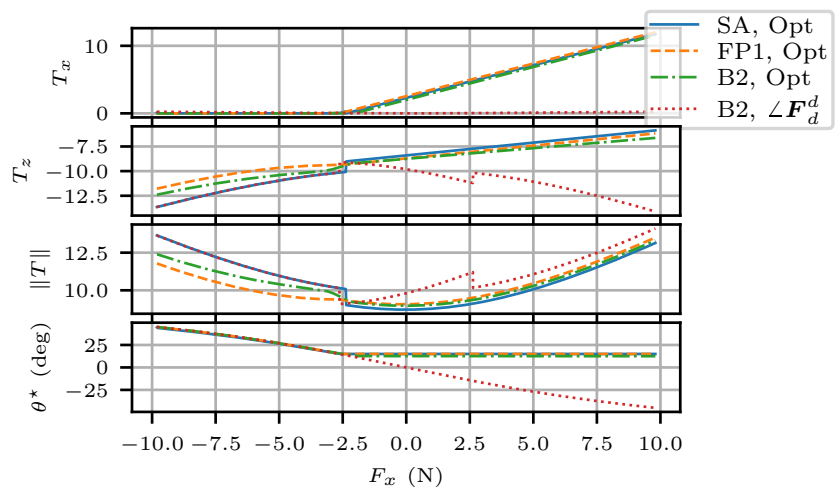

Fig. 7. Optimal thrust and pitch for increasing $F_{x}$, which makes $\boldsymbol{F}_{d}^{d}=$ $\left[F_{x}, 0,-m g\right]^{T}$. The other inputs to algorithm 1 are held constant at $V_{a}=$ $3.0 \mathrm{~m} / \mathrm{s}$ and $\gamma=0^{\circ}$.

and (14) over our previous nonlinear optimization approach we incorporate each into the control loop shown in fig. 1 and simulate tracking the trajectory used in [1]. The trajectory is meant to simulate a takeoff and landing in a congested area and is $100 \mathrm{~m}$ long, has a maximum altitude of $10 \mathrm{~m}$, a maximum velocity of $6.4 \mathrm{~m} / \mathrm{s}$, and includes a $90^{\circ}$ change in yaw. The simulation environment, described in [5], uses the blended 2 aerodynamic model as the true vehicle lift aerodynamics and the small-angle model for the true vehicle drag aerodynamics.

\section{A. Restricting the Pitch Rate}

Because algorithm 1 is solved at a single instant, the result may be discontinuous over successive iterations of the control loop. To avoid rapid changes in the pitch rate, we restrict the rate of change of the pitch command. Letting $\theta^{\star}$ be the solution from algorithm 1 , and $\theta_{\text {prev }}$ be the previously commanded pitch angle, we pick the commanded pitch angle to be

$$
\theta_{p / d}= \begin{cases}\theta_{\text {prev }}+\Delta t \omega_{y_{\max }} & \theta^{\star}-\theta_{\text {prev }}>\Delta t \omega_{y_{\max }} \\ \theta_{\text {prev }}-\Delta t \omega_{y_{\max }} & \theta^{\star}-\theta_{\text {prev }}<-\Delta t \omega_{y_{\max }} \\ \theta^{\star} & \text { Otherwise }\end{cases}
$$

where $\Delta t$ is the time between iterations of the control loop and $\omega_{y_{\max }}$ is the maximum desired angular rate about the $\boldsymbol{y}_{d}^{p}$ axis. Once $\theta_{p / d}$ has been determined, we compute the commanded thrust $\boldsymbol{T}_{d}^{p}$ using eq. 12.

\section{B. Simulation Results}

Implementing the algorithm described above significantly improved the behavior of our controller in [1]. Figures 8 and 9 show the results of the nonlinear optimization method (N Opt.) from our previous work, applied to the blended 2 model; the $\angle \boldsymbol{F}_{d}^{d}$ (multirotor-like flight) method; and the new sampling method that is described here, applied to the small-angle, flat plate 1 , and blended 2 aerodynamic models. Table II shows the error and thrust averaged along the trajectory for each of the different pitch allocation results. The sampling method on all three models had a similar average error and average thrust. The nonlinear optimization method 


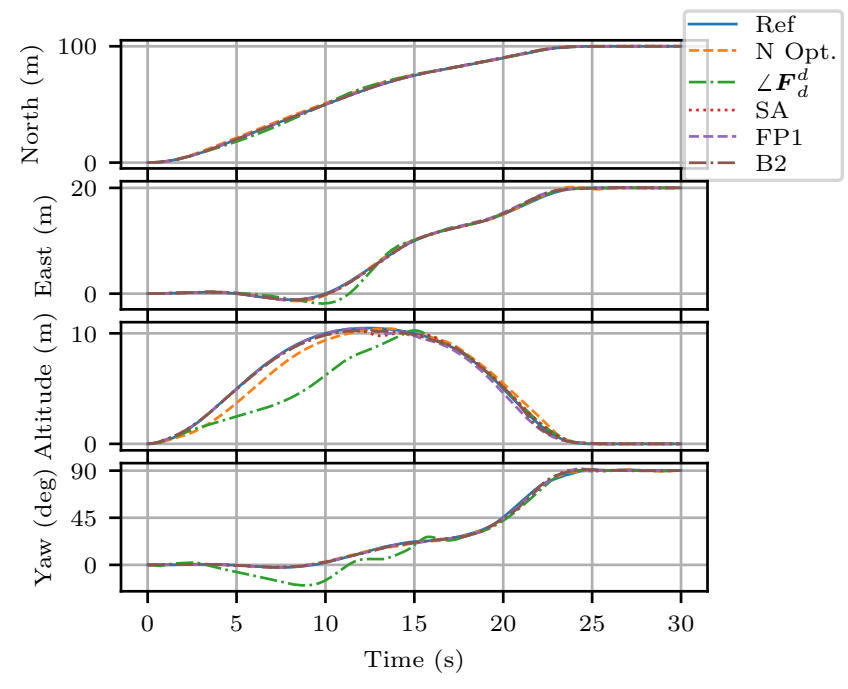

Fig. 9. Reference and actual positions for the trajectory used by fig. 8

TABLE II

SIMULATION AVERAGE POSITION ERROR AND THRUST

\begin{tabular}{lcc}
\hline Method & Avg. error (m) & Avg. thrust (N) \\
\hline Nonlinear Optimizer on B2 & 0.830 & 8.86 \\
Multirotor & 1.46 & 10.3 \\
Small-Angle & 0.188 & 9.01 \\
Flat Plate 1 & 0.22 & 9.03 \\
Blended 2 & 0.17 & 8.94
\end{tabular}

shows a slightly lower average thrust than the sampling methods, but it experiences a significantly higher average error. We attribute this to the optimization getting stuck in a local minimum. All of the pitch and thrust allocation methods significantly outperform the multirotor-like controller for this trajectory.

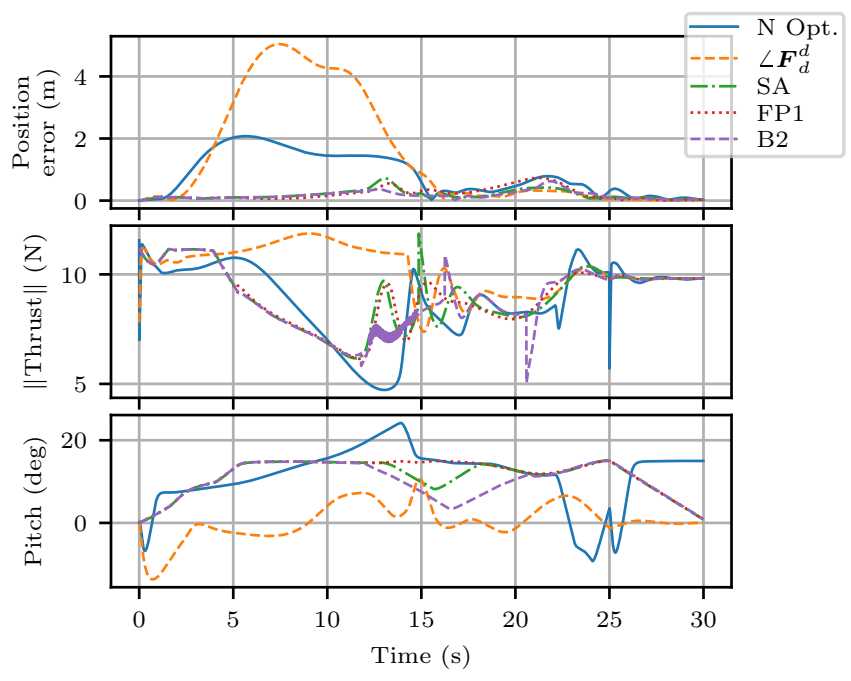

Fig. 8. Comparison of position error, thrust magnitude, and pitch angle for a sample trajectory including takeoff, cruise, and landing.

\section{CONClusion}

Trajectory tracking control of a winged eVTOL can be improved by considering the high angle-of-attack aerodynamics experienced during navigational flight. Using our global sampling-based algorithm to find the optimal pitch on an aerodynamic model that approximates the lift and drag experienced at high attack angles significantly reduces the tracking error of our previously presented trajectory tracking controller. This algorithm can also be applied to aerodynamic models derived from experimental data or to models that include flow separation.

The results presented here are a step towards the application of our proposed trajectory tracking controller to winged eVTOL UAV flight hardware. Additional future work includes identifying and approximating the high-angle-ofattack aerodynamic model for a flight vehicle.

\section{REFERENCES}

[1] J. B. Willis and R. W. Beard, "Nonlinear trajectory tracking control for winged eVTOL UAVs," in 2021 American Control Conference (ACC), 2021.

[2] A. Anglade, J. Kai, T. Hamel, and C. Samson, "Automatic control of convertible fixed-wing drones with vectorized thrust," in 2019 IEEE 58th Conference on Decision and Control (CDC), 2019, pp. 58805887.

[3] T. Lee, M. Leok, and N. H. McClamroch, "Geometric tracking control of a quadrotor UAV on SE(3)," in 49th IEEE Conference on Decision and Control (CDC), 2010, pp. 5420-5425.

[4] L. Meier, D. Honegger, and M. Pollefeys, "PX4: A node-based multithreaded open source robotics framework for deeply embedded platforms," in 2015 IEEE international conference on robotics and automation (ICRA). IEEE, 2015, pp. 6235-6240.

[5] J. Willis, J. Johnson, and R. W. Beard, "State-dependent LQR control for a tilt-rotor UAV," in 2020 American Control Conference (ACC), 2020, pp. 4175-4181.

[6] R. Cory and R. Tedrake, "Experiments in fixed-wing UAV perching," in AIAA Guidance, Navigation and Control Conference and Exhibit, 2008, p. 7256.

[7] D. Pucci, T. Hamel, P. Morin, and C. Samson, "Nonlinear feedback control of axisymmetric aerial vehicles," Automatica, vol. 53, pp. 7278, 2015.

[8] R. F. Stengel, Flight Dynamics. Princeton University Press, 2015.

[9] J. L. Moore, "Powerline perching with a fixed-wing UAV," Ph.D. dissertation, Massachusetts Institute of Technology, 2011.

[10] W. Pointner, G. Kotsis, P. Langthaler, and M. Naderhirn, "Using formal methods to verify safe deep stall landing of a MAV," in 2011 IEEE/AIAA 30th Digital Avionics Systems Conference. IEEE, 2011, pp. 5D1-1.

[11] R. W. Beard and T. W. McLain, Small Unmanned Aircraft: Theory and Practice. Princeton University Press, 2012.

[12] K. Gryte, "High angle of attack landing of an unmanned aerial vehicle," Master's thesis, NTNU, 2015.

[13] D. V. Uhlig and M. S. Selig, "Modeling micro air vehicle aerodynamics in unsteady high angle-of-attack flight," Journal of Aircraft, vol. 54, no. 3, pp. 1064-1075, 2017.

[14] M. Goman and A. Khrabrov, "State-space representation of aerodynamic characteristics of an aircraft at high angles of attack," Journal of Aircraft, vol. 31, no. 5, pp. 1109-1115, 1994. 